New criteria of asymmetry of rhythmic activities (alpha, beta etc.) were proposed :

A voltage decrease, if shifting, but predominantly unilateral and confirmed in two records of different lead montages, was considered "relatively ab. normal" when associated with 1) a more irregular morphology and 2) reduction of continuity.

The "relatively abnormal EEG sign" was regarded as highly significant in predicting existence of hematoma, if clinical correlates, such as symptoms of elevated intracranial pressure, mental deterioration, contralateral motor weakness and a history of head injury several months before.

The "relatively abnormal sign" was confirmed by retrospective EEG studies including several cases of verified hematoma, cases of meningioma and ventricle tumor. Six out of seven surgically proven hematoma were exactly anticipated, and in one angiographically suspected case, absence of hematoma was correctly predicted.

\title{
96. Clinical Observations on Subdural Hematoma with a Special Reference to their Occurrence
}

\author{
Tetsuhiko Asakura, Kunio Shinzato, Masayuki Atsuchi, \\ Yozi Yokoyama*, Motoi Nishimura and Motoi Kurorwa** \\ Department of Neurology* and Surgery**, Kagoshima University School of Medicine
}

Nine cases of subdural hematoma were experienced in recent three years. Seven were male and two were female. Age distributions were from fourteen to seventy years (Average 32 years old).

Eight cases of them were traumatic and the intervals between the trauma and the first visit to our clinic were from one to three months (Average 2 months). The trauma was produced by misfortune in the daily life of seniles (2), quarrel (2), traffic accident (2), sport playing (1) and misfortune in the daily life of youth (1). Chief complaints were headache (6 cases), nausea (5), vomiting (4), disturbance of consciousness (2), dizziness (1), sleep disturbance (1), gait disturbance (1), diplopia (1) and hemiplegia (1). Anisocoria and high blood pressure were marked in three cases. Seven cases showed increased deep reflexes on the contralateral side of hematoma, which placed on the left side in all cases.

EEG findings were characteristic in each cases, d.i. diffuse irregular slow activities with some laterality (3); without laterality (2), dysrhythmia 
(1), slow wave focus (1) and paroxysmal dysrhythmia with interval electrical silence (1).

Two cases took unfortune courses. One died of ventricular flatter during the surgery. The other died ten days after the removal of hematoma by a osteoplastic craniotomy and was autopsied. There were found of multiple intracerebral hematomas and sclerosing processes of the cerebral arteries.

Survival cases showed EEG abnormalities for long time.

In the non-traumatic cases ( 8 years old girl) complained of enlarging hyperostosis of the skull vault, there was found of a varix ruptured into the subdural space through the arachnoid. She died of the increased intracranial pressure in spite of performance of decompressive procedure four days after the removal of the hematoma and the ligature of the varix.

From the above described clinical courses, postoperatively long-lasting EEG abnormalities, surgery and autopsy findings, it was seemed to be some dispositional problems in the occurrence of subdural hematoma among the large number of head injury.

\title{
97. Dural Plasty with Tetoron Mesh: its Clinical and Experimental Studies
}

\author{
Yoshikazu SaIto \\ 2nd Surgical Department, Tottori University School of Medicine
}

The treatment with dural plasty in nine cases including three children were reported in the present study. They were all operated on for dural defect by utilizing Tetoron-Mesh. The clinical diagnosis of them were head injury (6), parasagittal meningioma (1), craniostenosis (1) and metastatic brain tumor from pulmonary cancer (1).

Results of operative procedure were all well except one case of head injury who was reoperated on because of the leakage of cerebrospinal fluid from insufficient suture defects. In one case of a metastatic brain tumor, the repaired mesh specimen was removed after death revealing no adhesion.

Experimental study was done using adult dogs having artificial dural defects which were repaired with Tetron mesh. Histological findings of repaired artificial dura was as follows:

1. After ten days Tetoron mesh was covered already with granulation tissues having moderate inflammatoric reactions which was decreased gradually in the following stadium. 